\title{
Intranasal Borneol Improves the Behavioral Problems and Enhances the Immunologic Function in Children with Autism
}

\section{Han YMY', Sze SL ${ }^{2,3}$, Wong $\mathrm{CK}^{4,5}$, and Chan $\mathrm{AS}^{2,3,6}$}

${ }^{1}$ Department of Rehabilitation Sciences, The Hong Kong Polytechnic University, Hung Hom, Kowloon, Hong Kong SAR

${ }^{2}$ Neuropsychology Laboratory, Department of Psychology, The Chinese University of Hong Kong, Shatin, Hong Kong SAR

${ }^{3}$ Chanwuyi Research Center for Neuropsychological Well-Being, The Chinese University of Hong Kong, Shatin, Hong Kong SAR

${ }^{4}$ Department of Chemical Pathology, The Chinese University of Hong Kong, Prince of Wales Hospital, Shatin, Hong Kong SAR

${ }^{5}$ Institute of Chinese Medicine and State Key Laboratory of Phytochemistry and Plant Resources in West China, The Chinese University of Hong Kong, Shatin, Hong Kong SAR

${ }^{6}$ Henan Songshan Research Institute for Chanwuyi, Henan 452470, China

\begin{abstract}
Children with autistic spectrum disorder (ASD) have difficulty interacting with others both verbally and behaviorally In addition, they display impaired temper and behavior control. These two impairments hamper the daily functions required for independent living. The current study evaluated a patented intranasal herbal formula including borneol as a possible intervention for improving the social ability and self-control of children with ASD. Thirty children with ASD participated in this study, with 15 receiving the herbal medicine and 15 serving as control subjects. After six months of intervention, the children in the experimental group showed significant improvements in their social and self-control abilities. Furthermore, their immunologic function, as indexed by the significant reduction in circulating CD3+CD8+ suppressor/cytotoxic T lymphocytes in their blood samples, was significantly improved. By contrast, the children in the control group did not show improvement in their social or self-control abilities, and no significant difference was found in their immunologic function over the six-month period. Thus, the results suggest that the intranasal herbal formula has therapeutic effects on the behavioral problems of children with ASD.
\end{abstract}

Keywords: Intranasal; Herbal remedy; Borneol; Autism spectrum disorder; Social behavior; Inhibition; Immunology; Lymphocytes

\section{Abbreviations}

ABC-L: Lethargy Subscale of the Aberrant Behavior Checklist; ADI-R: Autism Diagnostic Interview-Revised; ASD: Autism Spectrum Disorder; ATEC-I: Communication Subscale of the Autism Treatment Evaluation Checklist; ATEC-II: Sociability Subscale of the Autism Treatment Evaluation Checklist; CD3+CD8+: Suppressor/Cytotoxic T Lymphocytes; CD8+CTLs: Cytotoxic T Lymphocytes; CNS: Central Nervous System; CPRS-H: Children's Psychiatric Rating Scale; CRSR-H: Conners' Rating Scales-Revised

\section{Introduction}

Intranasal delivery has emerged as a potential intervention method for various neurological disorders [1]. Studies have suggested that this method provides a non-invasive option for administering drugs or substances that cannot be delivered across the blood-brain barrier. Empirical studies on intranasal administration for various brain disorders have examined the effects of benzodiazepines on epilepsy [2,3], naloxone on eating disorders [4], stem cells on Parkinson's disease and Huntington's disease [5,6], and insulin on Alzheimer's disease $[7,8]$. Research attention has recently focused on the effect of intranasal administration of oxytocin, a neuropeptide that is associated with the development of prosocial behavior [9], on the social behaviors of individuals with autistic spectrum disorder (ASD). While positive results have been reported [10-13], some treatment studies have found no significant difference between oxytocin and placebo administration on primary measures of social functioning and repetitive behaviors $[14,15]$. Authors have proposed that the differing phenotypes and etiologies of the subsets of ASD may account for the differences in the outcomes of the treatment studies.

Nasal oxytocin may have therapeutic effects for certain subgroups of individuals with ASD who have functional problems of the oxytocinergic system [16]. Furthermore, evidence increasingly suggests that intranasal administration is safe and effective $[1,17,18]$. Our laboratory has studied this delivery route with a herbal formula as an intervention to improve brain function. The herbal formula was developed based on the traditional Shaolin Chan-based medical principle. In Chan medicine, the intranasal administration of the herbal formula is one of the intervention techniques used to improve physical and psychological health problems [19]. The formula was recently patented as a method for improving brain function and as an intervention for brain disorders (Patent No.: ZL 20081 0176088.7). An active ingredient in the formula is natural borneol, which is well known in traditional Chinese medicine to improve the mind and thought processes.

In a pilot study [20], 12 children with a diagnosis of ASD were randomly assigned to either receive a daily administration of the herbal formula or a daily administration of a saline solution for one month. The treatment effect was determined by parental report, and the parents were blinded to the group assignment. Children who received the herbal formula displayed a significantly greater improvement

*Corresponding author: Agnes S Chan, Department of Psychology, The Chinese University of Hong Kong, Shatin, N.T., Hong Kong SAR, Tel: (852) 39436654; Fax: (852) 2603-5019; E-mail: aschan@psy.cuhk.edu.hk

Received October 26, 2015; Accepted November 26, 2015; Published November 30,2015

Citation: Han YMY, Sze SL, Wong CK, and Chan AS (2016) Intranasa Borneol Improves the Behavioral Problems and Enhances the Immunologic Function in Children with Autism. J Pharmacogn Nat Prod 2: 114 doi:10.4172/2472-0992.1000114

Copyright: (c) 2016 Han YMY, et al. This is an open-access article distributed under the terms of the Creative Commons Attribution License, which permits unrestricted use, distribution, and reproduction in any medium, provided the original author and source are credited. 
in spontaneous speech output, inhibition of repetitive speech, and initiation of behavior. Thus, these pilot data show promising results. The purpose of the present study was to examine the longer-term effects (i.e., six months) of this herbal formula on the behavior problems of children with ASD. We hypothesized that compared to the control group; children in the experimental group would demonstrate greater improvements in sociability, self-control, and flexible thinking, which are the core features of ASD.

In addition, increasing evidence has shown that there is an association between immune functions and the selective cognitive and behavioral symptoms in ASD [21]. Indeed, our previous study [22] showed that ASD children with lower functioning displayed higher levels of total $\mathrm{T}$ lymphocytes (CD3+) and suppressor/cytotoxic $\mathrm{T}$ lymphocytes (CD3+ $\mathrm{CD} 8+)$ and a higher percentage of suppressor/ cytotoxic T lymphocytes (CD3+ CD8+/CD 45+). These indices were also negatively correlated with measures of various cognitive functions and repetitive stereotyped behavior in ASD. These findings are in line with reports of immunologic abnormalities and detection of antiCNS auto-antibodies in children with ASD [23]. Of which, previous authors have speculated that the cytotoxic abnormalities of children with ASD are associated with neuronal damage [23-25], which leads to cognitive function deficits and abnormal behaviors in these children [26]. Interestingly, oxytocin has also been implicated to act as a link in the network between the immune, the endocrine, and the central nervous systems [27]. Since the therapeutic effects of the herbal formula have been postulated to parallel the effects of oxytocin, to understand the mechanisms underlying the potential treatment effects of the intranasal herbal formula on the documented altered immune function of children with ASD, several immune function indices were measured.

\section{Materials and Method}

\section{Participants}

Forty-two children with ASD, aged between 7 and 17 years old, voluntarily participated in the study. The children were recruited from the database of the Neuropsychology Laboratory of the Chinese University of Hong Kong and from two local schools in Hong Kong. All children had received a diagnosis of Autistic Disorder, Asperger's Disorder, or Pervasive Developmental Disorder, Not Otherwise Specified based on the DSM-IV-TR criteria [28]. The severity of their autistic symptoms was assessed by a clinical psychologist based on the Autism Diagnostic Interview-Revised (ADI-R) [29]. The interview included detailed questions about the child's early development and current functioning. Four children declined to participate before the first assessment, and six children were excluded from the study because of the presence of other neurodevelopmental, psychiatric, or neurological comorbidities or the use of psychiatric medication. The study was conducted in accordance with the Helsinki Declaration of the World Medical Association Assembly. The research protocol was approved by the Joint Chinese University of Hong Kong-New Territories East Cluster Clinical Research Ethics Committee (CREC Ref. No.: CRE-2008.421-T) and the Chinese Clinical Trial Registry (Registration No.: ChiCTR-TRC-12001857). Written consent was obtained from the parent of each participant prior to the study.

Of the remaining 32 children, 15 children served as the experimental group and received the six-month nasal herbal formula. The other 17 children served as the control group. At the six-month follow-up assessment, two of the children from the control group dropped out of the study. Table 1 shows that the children in the two groups were matched on age $(t(28)=0.29, P=0.77)$, gender $\left(\chi^{2}(1)=0.24, P=0.62\right)$, types of diagnosis $(\chi 2(1)=2.69, \mathrm{P}=0.26)$ and the severity of autistic symptoms, as measured by the three ADI-R subscales, with $t$ statistics ranging from -1.43 to 0.74 and $P$-values ranging from 0.17 to 0.47 . The two groups also demonstrated comparable levels of general intelligence $(t(28)=-0.10, P=0.92)$.

\section{Herbal nasal drop}

The patented herbal nasal drop was manufactured by the Hong Kong Institute of Biotechnology Limited, which is controlled by the council of the Chinese University of Hong Kong. The product was manufactured under strict Good Manufacturing Practice (GMP) standards. The product was tested for levels of heavy metal and toxic elements, microbial examination, and pesticide residue. The testing results met the product safety guidelines of the Department of Health of the Government of Hong Kong. The major active ingredients include natural borneol and borax (please refer to the patent record for details). All ingredients in the formula are within the recommended daily dosages that are prescribed in Chinese Herbal Medicine, which is published by the Educational Board of the National Drug Administration. Blood samples were collected for each participant in the experimental group in the first and sixth month following the administration of the herbal formula, to test their liver functions for any adverse effects associated with the formula. Results showed that liver function indices for all participants of the experimental group were within normal range.

\section{Procedures}

Prior to the baseline assessment, all of the children and their parents were briefed on the procedure of the study, and informed consent was obtained from the parents. A clinical psychologist assessed each child's developmental and medical history based on a structured clinical interview with the parents and the child's daily behaviors based on the parents' ratings on four standardized questionnaires. Using venipuncture at a medical clinic, a registered nurse then drew $4 \mathrm{ml}$ of EDTA blood from each child. The blood samples were stored in a thermally insulated bag and transported to a clinical laboratory, where a laboratory technician performed blood assays. The clinical psychologist who conducted the interview, the nurse who drew the blood, and the technician who performed the blood assays were blinded to the rationale of the study and the group assignment.

Following the baseline assessments, the children in the experimental group were instructed to apply the herbal nasal drop at a dose of $10 \mathrm{ml}$ per day, whereas the children in the control group did not receive any treatment. Drug compliance was checked with a log book. Following the six-month treatment, the children's parents were assessed with the same set of questionnaires and the peripheral blood samples were collected from each child.

\section{Self-control ability}

The children's ability to control themselves was measured using two standardized rating scales, the Hyperactivity subscales of the Children's Psychiatric Rating Scale (CPRS-H) [30] and the Conners' Rating Scales-Revised (CRS-R-H) [31]. These two subscales were selected as measures of inhibitory control of behavior and emotion. The CPRS-H rates four self-control aspects on a scale from 0 to 6 , and the CRS-R-H rates 10 disinhibition-related items on a scale from 0 to 3. The total subscale scores were computed separately for comparison, with higher scores indicating poorer inhibitory control. 
Citation: Han YMY, Sze SL, Wong CK, and Chan AS (2016) Intranasal Borneol Improves the Behavioral Problems and Enhances the Immunologic Function in Children with Autism. J Pharmacogn Nat Prod 2: 114. doi:10.4172/2472-0992.1000114

Page 3 of 7

\begin{tabular}{|c|c|c|c|c|}
\hline Characteristics & $\begin{array}{l}\text { Control Group } \\
\qquad(n=15)\end{array}$ & $\begin{array}{l}\text { Experimental Group } \\
\qquad(n=15)\end{array}$ & $t / X^{2}$ & $P$ value \\
\hline Age (mean $\pm S D$ ), years & $11.60 \pm 2.64$ & $11.88 \pm 2.70$ & 0.29 & 0.77 \\
\hline Gender-Male (\%) & 80 & 86.7 & 0.24 & 0.62 \\
\hline IQ (mean $\pm S D)$ & $85.25 \pm 15.98$ & $84.53 \pm 23.60$ & -0.1 & 0.92 \\
\hline \multicolumn{3}{|l|}{ Diagnosis } & 2.69 & 0.26 \\
\hline Autistic Disorder (\%) & 66.7 & 40 & - & - \\
\hline PDD-NOS (\%) & 33.3 & 53.3 & - & - \\
\hline Asperger's Disorder (\%) & 0 & 6.7 & - & - \\
\hline \multicolumn{5}{|l|}{ Severity of Disorder (mean $\pm \mathrm{SD}$ ) } \\
\hline ADI-R Social Interaction & $19.20 \pm 6.73$ & $21.07 \pm 7.15$ & 0.74 & 0.47 \\
\hline ADI-R Communication & $14.20 \pm 4.30$ & $11.93 \pm 4.42$ & -1.43 & 0.17 \\
\hline ADI-R Stereotyped Behavior & $4.73 \pm 2.81$ & $3.93 \pm 2.91$ & -0.77 & 0.45 \\
\hline
\end{tabular}

Note: $A D I-R=A u t i s m$ Diagnostic Interview-Revised; $I Q=$ Intelligence quotient as assessed by the Chinese version of Wechsler Intelligence Scale for Children-Third Edition or the Stanford-Binet Intelligence Scale-Fourth Edition; PDD-NOS=Pervasive Developmental Disorders, Not Otherwise Specified.

Table 1: Baseline demographic and clinical characteristics of participants in the control and experimental groups.

\section{Sociability}

The children's ability to interact with other people was measured by the Lethargy subscale of the Aberrant Behavior Checklist (ABC-L) [32] and the Sociability subscale of the Autism Treatment Evaluation Checklist (ATEC-II) [33] These subscales measure the degree of appropriate forms of social behavior (e.g., awareness of the surrounding environment and the initiation of an appropriate social response while inhibiting inappropriate responses). The ABC-L comprises 16 items that are rated from 0 to 3 , and the ATEC-II comprises 20 items that are rated from 0 to 2 . The total subscale scores were computed separately, with higher scores indicating greater deficits in social behaviors.

\section{Communication ability}

The children's ability to comprehend instructions and communicate verbally was assessed using the Communication subscale of the ATEC (ATEC-I) [33]. This subscale comprises 14 items that are rated from 0 to 2 , with higher scores indicating greater verbal communication problems.

\section{Immunological measures}

Peripheral blood mononuclear cells (PBMC) were prepared by centrifugation of blood using a density gradient (Ficoll-Paque Plus, Amersham Pharmacia Biotech Ltd., Uppsala, Sweden). Cell surface expression of total lymphocytes (CD45+), suppressor/ cytotoxic $\mathrm{T}$ lymphocytes (CD3+CD8+), and $\mathrm{T}$ helper lymphocytes $(\mathrm{CD} 4+)$ in the children's peripheral blood was measured using an immunofluorescence technique with the BD Multitest IMK kit using flow cytometry (FACSCalibur 4 color flow cytometer, BD Bioscience Corp., San Jose, CA, USA). The percentage of suppressor/cytotoxic $\mathrm{T}$ lymphocytes $(\mathrm{CD} 3+\mathrm{CD} 8+)$ in proportion to the total lymphocytes (CD45+) and the T-helper/suppressor ratio were computed for subsequent comparisons.

\section{Data analyses}

An intention-to-treat analysis was performed to compare the prepost scores of the parent-rated problem behaviors for each group of children using repeated measures ANOVA followed by post hoc paired $t$ tests. The changes in immunological indices were compared using paired $t$ tests, and their association with the changes in behavioral measures was explored with Pearson's correlation analyses. The analyses were conducted using the software SPSS. Given that specific hypotheses were tested, no adjustment to the alpha level was applied, which avoided reducing the power of the tests. The effect sizes (E.S.) and $95 \%$ confidence intervals (C.I.) were used to evaluate the extent of pre-post changes in each group.

\section{Results}

\section{Intranasal herbal formula improved self-control ability in children with ASD}

As shown in Figure 1a, prior to the treatment, the parents' ratings on the CPRS-H and CRS-R-H did not significantly differ between the control and experimental groups $(t=0.191$ and $-0.155, P=0.850$ and 0.878 , respectively). A comparison between the pre- and post-treatment ratings using repeated measures ANOVA showed a significant main effect of time (pre-score vs. post-score) on the CPRS-H score $(F=8.645, P=0.007)$ and a marginally significant time by group (control vs. treatment) interaction effect on the CRS-R-H score $(F=3.965$, $P=0.056)$. According to the post hoc $t$ tests, following the treatment, the experimental group demonstrated a significant decrease in the CPRS-H score $(t=2.714, P=0.009$, E.S. $=0.701$, 95\% C.I. $=0.713-6.087)$, whereas the control group did not display a significant difference $(t=1.356, P=0.099$, E.S. $=0.350,95 \%$ C.I. $=-0.853-3.787)$. Similar results were obtained from the CRS-R-H score. Following the treatment, the control group did not show a significant difference in the CRS-R-H score $(t=-0.555, P=0.294$, E.S. $=0.143$, $95 \%$ C.I. $=-3.568-2.102)$, whereas the experimental group demonstrated a significant decrease $(t=2.467$, $P=0.014$, E.S. $=0.636,95 \%$ C.I. $=0.348-4.985$ ). These results suggest that the nasal herbal formula improves ASD children's self-control ability.

\section{Intranasal herbal formula improved sociability in children with ASD}

Prior to the treatment, the parent-rated sociability subscales of the ABC-L and ATEC were comparable between the groups $(t=0.469$ and $0.030, P=0.643$ and 0.977 , respectively; Figure $1 \mathrm{~b}$ ). Following the treatment, the pattern of changes in sociability was comparable to that of children's self-control ability. The repeated measures ANOVA found a significant time (pre-score vs. post-score) by group (control vs. treatment) interaction effect on the ABC-L score $(F=4.698, P=0.039)$, but there was no significant interaction on the ATEC-II score $(F=2.108$, $P=0.158$ ). Subsequent post hoc $t$ tests revealed that children who did not receive the treatment showed no significant pre- and post-difference in the ABC-L score $(t=0.423, P=0.678$, E.S. $=0.109,95 \%$ C.I. $=-2.439$ $3.639)$ or the ATEC-II score $(t=-0.223, P=0.826$, E.S. $=0.058,95 \%$ C.I. $=-4.241-3.441$ ). By contrast, children in the experimental group showed significant improvements in their social ability, which was 
Citation: Han YMY, Sze SL, Wong CK, and Chan AS (2016) Intranasal Borneol Improves the Behavioral Problems and Enhances the Immunologic Function in Children with Autism. J Pharmacogn Nat Prod 2: 114. doi:10.4172/2472-0992.1000114

demonstrated by their significantly reduced ABC-L ( $t=2.775, P=0.008$, E.S. $=0.717,95 \%$ C.I. $=1.500-11.700)$ and ATEC-II $(t=2.381, P=0.016$, E.S. $=0.615,95 \%$ C.I. $=0.265-5.068)$ scores.

\section{Intranasal herbal formula improved communication ability in children with ASD}

Figure 1c presents the parents' pre- and post-treatment ratings on the ATEC-I subscale, which measures children's communication ability. The baseline ratings were comparable between the groups $(t=-$ 1.310, $P=0.201$ ). Both the repeated measures ANOVA and the $t$ test results were consistent with the other rating scales and demonstrated that children in the experimental group showed significant

(A)
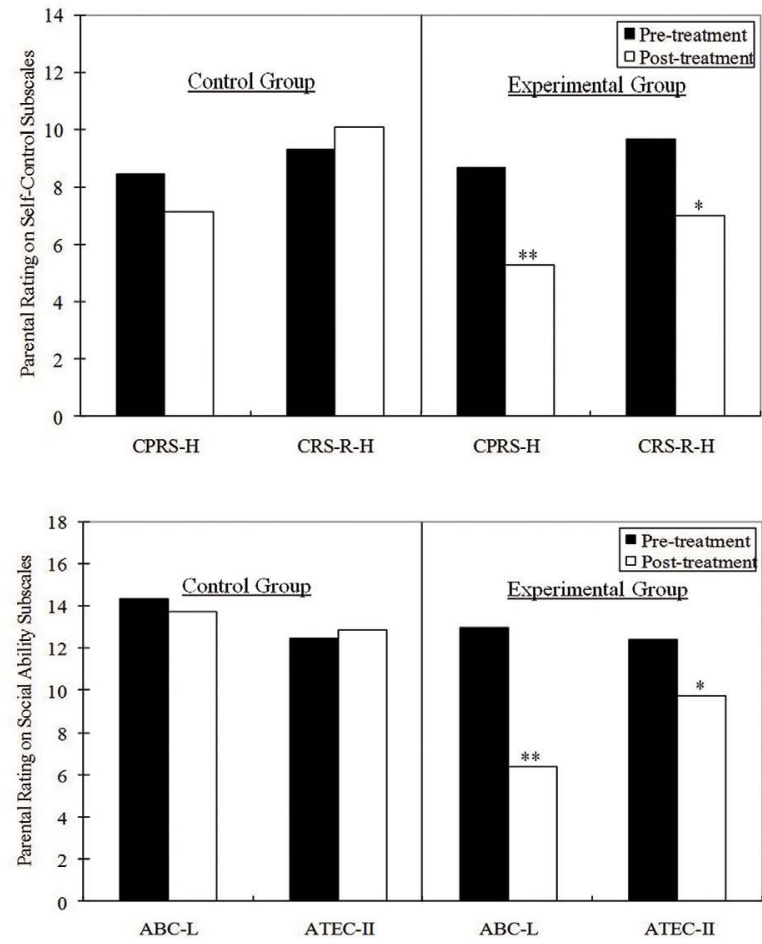

(c)

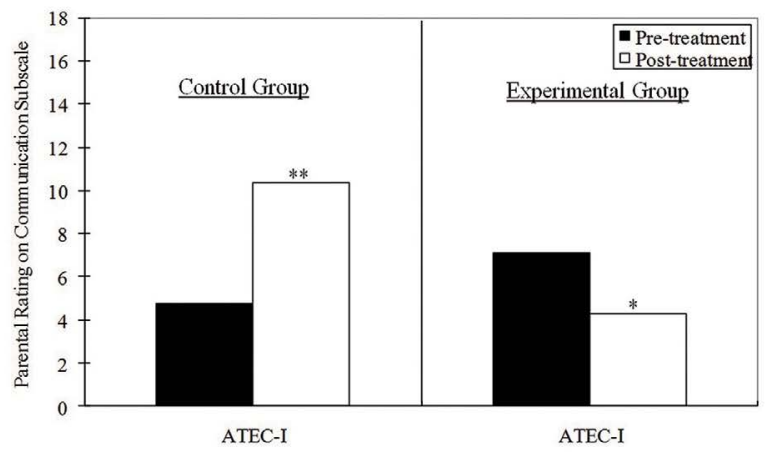

Figure 1: Parent ratings on standardized behavioral questionnaires that measure problems related to the (a) self-control, (b) social, and (c) communication abilities of their children at pre- and post-treatment. Higher scores indicate a greater degree of behavioral problems.

CPRS-H=Hyperactivity subscale of the Children's Psychiatric Rating Scale CRS-R-H=Hyperactivity subscale of the Conners' Rating Scale-Revised; $A B C-L=$ Lethargy subscale of the Aberrant Behavior Checklist; ATECII=Sociability subscale of the Autism Treatment Evaluation Checklist; ATEC $\mathrm{I}=$ Communication subscale of the Autism Treatment Evaluation Checklist. ${ }^{* *} p<0.01 ;{ }^{*} p<0.05$ improvements in communication ability, as measured by the ATEC-I $(F=34.502, P<0.001 ; t=2.432, P=0.015$, E.S. $=0.628,95 \%$ C.I. $=0.339$ 5.395). By contrast, the children in the control group showed reduced communication ability after six months $(t=-6.832, P<0.001$, E.S. $=1.765$, $95 \%$ C.I. $=-7.270--3.796$ ).

\section{Intranasal herbal formula improved immune function in children with ASD}

Prior to the treatment, there were no significant differences in the three selected $\mathrm{T}$ lymphocyte subsets between the control and experimental groups, with $t$ statistics ranging from -0.715 to 0.732 and $P$-values are ranging from 0.473 to 0.930 . As shown in Figure $2 \mathrm{a}$, the children in the experimental group displayed a significant reduction in the absolute cells count of suppressor/cytotoxic $\mathrm{T}$ lymphocytes $(\mathrm{CD} 3+\mathrm{CD} 8+)$. The experimental group showed a statistically significant reduction of 129 cells $/ \mu \mathrm{l}(t=2.066, P=0.029$, E.S. $=0.533$, 95\% C.I. $=$ 4.939-264.005), whereas the control group reduction of $98 \mathrm{cells} / \mu \mathrm{l}$ was not statistically significant $(t=1.910, P=0.053$, E.S. $=0.722$, 95\% C.I. $=$ 27.549-223.549). When considering the extent of change in proportion to the amount of total lymphocytes (CD45+), which is reflected by the percentage of suppressor/cytotoxic T lymphocytes (CD3+CD8+1 $\mathrm{CD} 45+)$, the experimental group showed a significant reduction of 1.13\% $(t=1.817, P=0.046$, E.S. $=0.469$, 95\% C.I. $=-0.205-2.471$; Figure $2 b)$. By contrast, the control group did not display a significant difference following the treatment $(t=-0.387, P=0.356$, E.S. $=0.146,95 \%$ C.I.=-4.187-3.044). Regarding the T-helper/suppressor ratio (CD4+/ CD8+), which is relatively lower in individuals with ASD [34], the experimental group demonstrated a significant increase of 0.097 units in the ratio $(t=-2.923, P=0.006$, E.S. $=0.757,95 \%$ C.I. $=-0.169--0.026)$. By contrast, the increase of 0.071 units in the control group was not statistically significant $(t=-1.266, P=0.126$, E.S. $=0.478,95 \%$ C.I. $=-$ 0.209-0.067; Figure 2c).

\section{Correlation between immune function indices and behavioral measures}

Given the significant changes in daily behavioral problems and immunological indices in the experimental group, we explored the potential linkage between the two parameters. Pearson's correlation analyses were performed between the pre-post differences in behavioral measures and $\mathrm{T}$ lymphocyte subsets (Table 2 ). The reduction in the absolute amount of suppressor/cytotoxic T lymphocytes (CD3+CD8+) was associated with a reduction in hyperactive behaviors, as measured by the CPRS-H $(r=0.719, P=0.002)$ and CRS-R-H $(r=0.491, P=0.032)$; the association was medium to large in strength. Furthermore, the increment in the T-helper/suppressor ratio (CD4+/CD8+) was significantly correlated with a reduction in hyperactive behaviors, as measured by the CRS-R-H ( $r=-0.497, P=0.030)$, and the increment was marginally correlated with a reduction in social problems, as measured by the ATEC-II $(r=-0.401, P=0.070)$. However, there was no significant linear association between the change in the percentage of suppressor/ cytotoxic $\mathrm{T}$ lymphocytes $(\mathrm{CD} 3+\mathrm{CD} 8+/ \mathrm{CD} 45+)$ and behavioral problems.

\section{Discussion}

The present study examined the treatment effects of daily administrations of an intranasal herbal remedy with borneol for six months on the social deficits and problem behaviors of children with ASD. The results showed that the herbal formula significantly improved the sociability, communication, and self-control ability of children with ASD. These children showed a significant reduction 
(A)

(B)
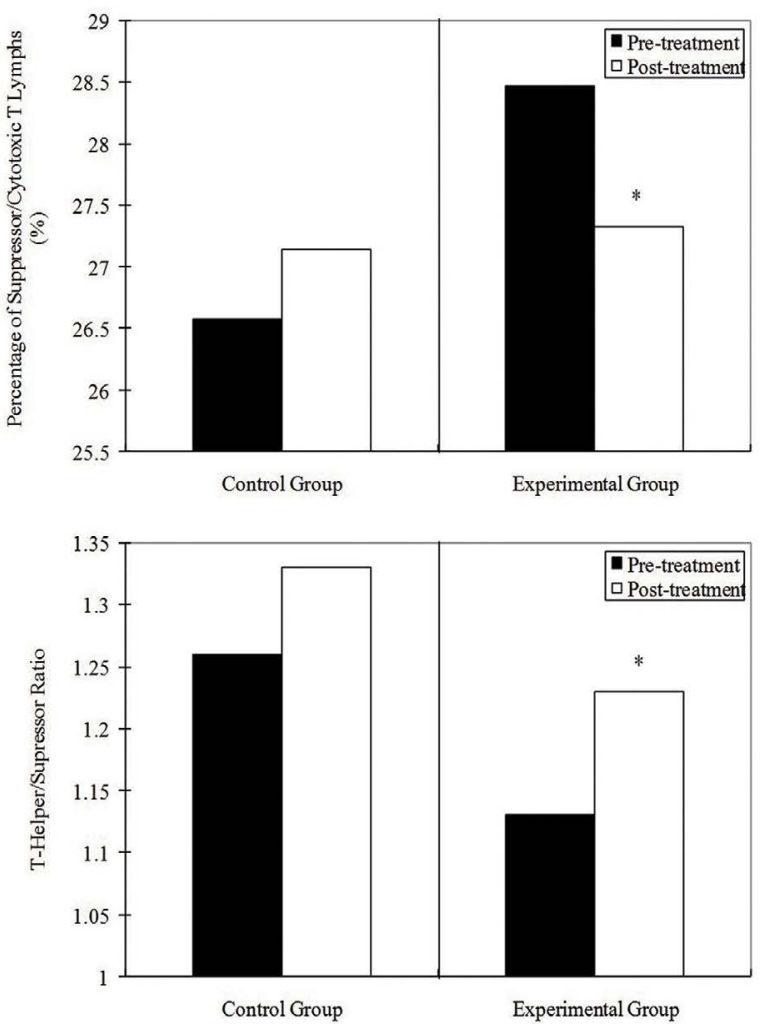

Figure 2: Levels of (a) absolute cells count of suppressor/cytotoxic T lymphocytes $(\mathrm{CD} 3+\mathrm{CD} 8+)$, (b) percentage of suppressor/cytotoxic T lymphocytes ratio $(\mathrm{CD} 3+\mathrm{CD} 8+/ \mathrm{CD} 45+)$, and (c) T-helper/suppressor ratio $(\mathrm{CD} 4+/ \mathrm{CD} 8+)$ at pre- and post-treatment.

${ }^{* *} p<0.01 ;{ }^{*} p<0.05$

in circulating $\mathrm{CD} 3+\mathrm{CD} 8+$ and an enhanced T-helper/suppressor ratio in their blood, both of which were associated with improved social functioning. By contrast, the control group of children with ASD did not show significant differences in measures of sociability and immunologic functioning over the six-month period. Thus, the results suggest that the significant improvement in social behavior and enhanced immunologic functioning are consequences of the therapeutic potential of repeated administrations of the intranasal herbal remedy with borneol rather than natural developmental growth.

Our findings are consistent with previous studies reporting that borneol may have anti-inflammatory [35], neuroprotective [36,37], and anti-oxidative DNA damage [38] effects. It has been suggested that because of borneol's ability to open and cross the blood-brain barrier over the hippocampus and hypothalamus regions, which are the areas of the brain that are most affected by central nervous system (CNS) disorders, borneol can assist the permeation of drugs that target the brain $[39,40]$. In our patent application, we reported that mice that received the herbal formula for seven days had higher levels $(p<0.001)$ of norepinephrine in the brain (mean: $46.10 \mathrm{pg} / \mathrm{mg}$ of tissue) than mice that received a saline solution (mean: $22.03 \mathrm{pg} / \mathrm{mg}$ of tissue). The mice that received three days of the herbal formula also displayed a higher level of norepinephrine in the brain (mean: approximately $33 \mathrm{pg} / \mathrm{mg}$ of tissue) than mice that received the saline solution $(\mathrm{p}<0.001)$. The preliminary data suggest that borneol may be an effective agent in improving brain activities.

Furthermore, in another pilot study with counterbalanced design [20], 14 adults were intranasally administered the herbal formula in one condition and a saline solution in a second condition. The neuro-electrophysiological activity of the anterior cingulate cortex was recorded using an electroencephalogram (EEG) and lowresolution brain electromagnetic tomography (LORETA). The results substantiated the effects of the herbal medicine on mice and showed that the administration of the herbal formula increased the activity of the anterior cingulate cortex; the saline solution had no such effect. Nevertheless, while the underlying neural mechanism of this herbal formula remains largely unknown, the therapeutic effects of the herbal formula show parallels to the effects of intranasal oxytocin as a method of improving social behavior in individuals with ASD. Studies of healthy adults have shown that oxytocin promotes social attachments [41], improves emotion recognition [42], and reduces anxiety and endocrine responses to social stress [43]. There is evidence that a disturbed endocrine oxytocin system may contribute to the social impairments in individuals with ASD [11]. While some ASD studies have shown positive results, including reductions in repetitive behaviors [13] and improvements in social memory [12] and emotion processing [10], the findings have been inconsistent $[14,15]$. Given the genetic variations of the oxytocin system found in individuals with ASD [44], the therapeutic effects of oxytocin are likely specific to ASD subgroups that have functional problems in the oxytocin receptor system [16].

In addition, the current findings of a significantly lower level of circulating $\mathrm{CD} 3+\mathrm{CD} 8+$ and an enhanced $\mathrm{T}$ helper/suppressor ratio after six months of daily administrations of intranasal herbal formula suggest that the underlying neural mechanism of borneol is associated with altered immune function in the children with ASD. The present study also showed that the improved social functioning was significantly associated with immune function indices. These findings are consistent with previous studies that reported abnormalities in the immune system of children with ASD $[24,45]$ and the finding that a predominance of cytotoxic $\mathrm{T}$ lymphocytes (CD8+CTLs) may be involved in the pathogenesis that underlies the cognitive deficits and problem behaviors in individuals with ASD $[23,26]$. Specifically, CD8+CTLs have highly potent cytotoxic functions, and they are important effectors in several autoimmune and degenerative CNS diseases that can cause direct tissue damage to the CNS $[23,46]$. Recent studies have shown that executive function deficits and repetitive stereotyped behavior are exacerbated as a function of increased levels of CD3+CD8+ in children with ASD [26]. Thus, the current findings of a robust enhancement in immune function in the experimental group and its association with improved social and inhibitory control abilities are consistent with the existing scientific findings regarding immunological factors in the pathogenesis of ASD. 
Citation: Han YMY, Sze SL, Wong CK, and Chan AS (2016) Intranasal Borneol Improves the Behavioral Problems and Enhances the Immunologic Function in Children with Autism. J Pharmacogn Nat Prod 2: 114. doi:10.4172/2472-0992.1000114

Page 6 of 7

\begin{tabular}{|c|c|c|c|}
\hline Behavioral Measures & Suppressor/cytotoxic T lymphs & Suppressor/cytotoxic T lymphs \% & T-helper/suppressor ratio \\
\hline CPRS-H & $0.719^{\star *}$ & -0.236 \\
\hline CRS-R-H & $0.491^{\star}$ & -0.066 \\
\hline ABC-L & 0.116 & -0.212 & -0.420 \\
\hline ATEC-II & 0.148 & -0.091 \\
\hline ATEC-I & 0.060 & 0.112 \\
\hline
\end{tabular}

Note. CPRS-H=Hyperactivity subscale of the Children's Psychiatric Rating Scale; CRS-R-H=Hyperactivity subscale of the Conners' Rating Scale-Revised; ABC-L=Lethargy subscale of the Aberrant Behavior Checklist; ATEC-II=Sociability subscale of the Autism Treatment Evaluation Checklist; ATEC-I=Communication subscale of the ATEC* $p<0.05 ;{ }^{* *} p<0.01$ at one-tailed.

Table 2: Correlations between behavioral measures and T lymphocyte subsets in the experimental group $(n=15)$.

The present study has provided several interesting observations on the potential therapeutic effects of intranasal herbal formula with borneol on the sociability and inhibitory control abilities of children with ASD. Future research is necessary to substantiate the present findings and to examine the underlying neural mechanism of borneol. One limitation of the present study is that the generalizability of the findings may be limited, as the sample size was relatively small. Therefore, future studies with larger sample sizes are needed to further validate the effects of the herbal formula. A second limitation is that the present study adopted subjective questionnaires. Objective neuropsychological measures and neuro-electrophysiological measures should be adopted in future studies to further verify the effects of this herbal formula.

\section{Conclusion}

The present study suggests that the patented intranasal herbal formula has therapeutic effects on the behavioral problems of children with ASD. The study's targeted behaviors included self-control, sociability, and communication ability. In addition to measures of these behaviors, several indicators of immune system improved following the treatment, which suggests that the behavioral change may be related to children's improved immunologic condition.

\section{Acknowledgement}

This study was supported by a donation from Mr. Law Sau Wang to the Chinese University of Hong Kong. The authors especially thank Venerable Master Dejian of the Songshan Monastery. They are also thankful to the parents and the children who participated in this study. Appreciation is also extended to Debbie Yan, Lan He, Man-ying Mo, Queenie Wong, and Rex Wong for their efforts in data collection and data management.

\section{References}

1. Chapman CD, Frey WH, Craft S, Danielyan L, Hallschmid M, et al. (2013) Intranasal treatment of central nervous system dysfunction in humans. Pharmacol Res 30: 2475-2484.

2. Fişgin T, Gurer $Y$, Tezic T, Senbil N, Zorlu P, et al. (2002) Effects of intranasal midazolam and rectal diazepam on acute convulsions in children: Prospective randomized study. J Child Neurol 17: 123-126.

3. Holsti M, Dudley N, Schunk J, Adelgais K, Greenberg R, et al. ( 2010) Intranasal midazolam vs rectal diazepam for the home treatment of acute seizures in pediatric patients with epilepsy. Arch Pediatr Adolesc Med 164: 747-753.

4. McElroy SL, Guerdjikova Al, Mori N, O'Melia AM (2012) Current pharmacotherapy options for bulimia nervosa and binge eating disorder. Expert Opin Pharmacother 13: 2015-2026.

5. Bossolasco P, Cova L, Levandis G, Diana V, Cerri S, et al. (2012) Non-invasive near-infrared live imaging of human adult mesenchymal stem cells transplanted in a rodent model of Parkinson's disease. Int J Nanomedicine 7: 435-447.

6. Danielyan L, Schäfer R, von Ameln-Mayerhofer A, Bernhard F, Verleysdonk S, et al. (2011) Therapeutic efficacy of intranasally delivered mesenchymal stem cells in a rat model of Parkinson disease. Rejuvenation Res 14: 3-16.
7. Craft S, Baker LD, Montine TJ, Minoshima S, Watson GS, et al. (2012) Intranasal insulin therapy for Alzheimer disease and amnestic mild cognitive impairment: a pilot clinical trial. Arch Neurol 69: 29-38

8. Schiöth HB, Frey WH, Brooks SJ, Benedict C (2012) Insulin to treat Alzheimer's disease: Just follow your nose? Expert Rev Clin Pharmacol 5: 17-20.

9. Insel TR (2010) The challenge of translation in social neuroscience: A review of oxytocin, vasopressin, and affiliation behaviour. Neuron 65: 768-779.

10. Andari E, Duhamel JR, Zalla T, Herbrecht E, Leboyer M, et al. (2010) Promoting social behaviour with oxytocin in high-functioning autism spectrum disorders. Proc Natl Acad Sci USA 107: 4389-4394.

11. Guastella AJ, Einfeld SL, Gray KM, Rinehart NJ, Tonge BJ, et al. (2010) Intranasal oxytocin improves emotion recognition for youth with autism spectrum disorders. Biol Psychiatry 67: 692-694.

12. Hollander E, Bartz J, Chaplin W, Phillips A, Sumner J, et al. (2007) Oxytocin increases retention of social cognition in autism. Biol Psychiatry 61: 498-503.

13. Hollander E, Novotny S, Hanratty M, Yaffe R, DeCaria CM, et al. (2003) Oxytocin infusion reduces repetitive behaviours in adults with autistic and Asperger's disorders. Neuropsychopharmacology 28: 193-198.

14. Anagnostou E, Soorya L, Chaplin W, Bartz J, Halpern D, et al. (2012) Intranasa oxytocin versus placebo in the treatment of adults with autism spectrum disorders: A randomised controlled trial. Mol Autism.

15. Dadds MR, MacDonald E, Cauchi A, Williams K, Levy F, et al. (2013) Nasa oxytocin for social deficits in childhood autism: a randomized controlled trial. J Autism Dev Disord 44: 521-531.

16. Bartz JA, Zaki J, Bolger N, Ochsner KN (2011) Social effects of oxytocin in humans: Context and person matter. Trends Cogn Sci 15: 301-309.

17. Djupesland PG (2013) Nasal drug delivery devices: Characteristics and performance in a clinical perspective: a review. Drug Deliv Transl Res 3: 42-62.

18. Illum L (2003) Nasal drug delivery: Possibilities, problems and solutions. J Control Release 87: 187-198.

19. Chan AS (2010) The Shaolin Chanwuyi: A Chinese Chan Buddhism. Chanwuyi Publishing House Limited, Hong Kong.

20. Chan AS, Cheung MC, Sze SL, Leung WW, Shi D (2011) An Herbal Nasa Drop Enhanced Frontal and Anterior Cingulate Cortex Activity. Evid Based Complement Alternat Med

21. Ashwood P, Van de Water J (2004) Is autism an autoimmune disease? Autoimmunity Rev 3: 557-562.

22. Han YMY, Leung WWM, Wong CK, Lam JMK, Cheung MC, et al. (2011) Lymphocyte subset alterations related to executive function deficits and repetitive stereotyped behaviour in autism. Res Autism Spect Disord 5: 486494.

23. Neumann H, Medana IM, Bauer J, Lassmann H (2002) Cytotoxic T lymphocytes in autoimmune and degenerative CNS diseases. Trends Neurosci 25: 313-319.

24. Krause I, He X, Gershwin ME, Shoenfeld Y (2002) Brief report: immune factors in autism: a critical review. J Autism Dev Disord 32: 337-345.

25. Schirmer M, Goldberger C, Wurzner R, Duftner C, Pfeiffer K, et al. (2001) Circulating cytotoxic CD8+ CD28- T cells in ankylosing spodylitis. Arthritis Res 4: $71-76$. 
Citation: Han YMY, Sze SL, Wong CK, and Chan AS (2016) Intranasal Borneol Improves the Behavioral Problems and Enhances the Immunologic Function in Children with Autism. J Pharmacogn Nat Prod 2: 114. doi:10.4172/2472-0992.1000114

26. Han YMY, Chan AS, Sze SL, Cheung MC, Wong CK, et al. (2013) Altered immune function associated with disordered neural connectivity and executive dysfunctions: A neurophysiological study on children with autism spectrum disorders. Res Autism Spect Disord 7: 662-674.

27. Barnard A, Layton D, Hince M, Sakkal S, Bernard C, et al. (2008) Impact of the neuroendocrine system on thymus and bone marrow function. Neuroimmunomodulation 15: 7-18.

28. American Psychiatric Association (2000) Diagnostic and Statistical Manual of Mental Disorders. (4 $4^{\text {th }}$ edn) Washington (DC), USA

29. Lord C, Rutter M, Couteur AL (1994) Autism Diagnostic Interview-Revised: a revised version of a diagnostic interview for caregivers of individuals with possible pervasive developmental disorders. J Autism Dev Disord 24: 659-685.

30. Fish B (1985) Children's psychiatric rating scale. Psychopharmacol Bull 2: 753770 .

31. Conners CK (1997) Conners' Rating Scales-Revised. Multi-Health Systems, New York

32. Aman MG, Singh NN, Stewart AW, Field CJ (1985) The aberrant behavior checklist: A behavior rating scale for the assessment of treatment effects. Am J Ment Defic 89: 485-491.

33. Autism Treatment Evaluation Checklist (2000) Reliabilities and score distributions.

34. Gupta VB (2004) Etiology of autism: Autistic Spectrum Disorders in Children New York 40-59.

35. Juhás S1, Cikos S, Czikková S, Veselá J, ll'ková G, et al. (2008) Effects of borneol and thymoquinone on TNBS-induced colitis in mice. Folia Biol (Praha) $54: 1-7$

36. Chen XH, Lin ZZ, Liu AM, Ye JT, Luo Y, et al. (2010) The orally combined neuroprotective effects of sodium ferulate and borneol against transient global ischaemia in C57 BL/6J mice. J Pharm Pharmacol 62: 915-923.
37. Liu R, Zhang L, Lan X, Li L, Zhang TT, et al. (2011) Protection by borneol on cortical neurons against oxygen-glucose deprivation/ reperfusion: involvement of anti-oxidation and anti-inflammation through nuclear transcription factor kappa B signaling pathway. Neuroscience 176: 408-419.

38. Horváthová E, Kozics K, Srančíková A, Hunáková L, Gálová E et al. (2012) Borneol administration protects primary rat hepatocytes against exogenous oxidative DNA damage. Mutagenesis 27: 581-588.

39. Su J, Lai H, Chen J, Li L, Wong YS, et al. (2013) Natural borneol, monoterpenoid compound, potentiates selenocystine-induced apoptosis in human hepatocellular carcinoma cells by enhancement of cellular uptake and activation of ROS-mediated DNA damage. PLoS One.

40. Yu B, Ruan M, Dong X, Yu Y, Cheng H (2013) The mechanism of the opening of the blood-brain barrier by borneol: A pharmacodynamics and pharmacokinetics combination study. J Ethnopharmacol 150: 1096-1108.

41. 41. Donaldson ZR, Young LJ (2008) Oxytocin, vasopressin, and the neurogenetics of sociality. Science 322: 900-904.

42. Domes G, Heinrichs M, Michel A, Berger C, Herpertz S (2007) Oxytocin improves "mind-reading" in humans. Biol Psychiatry 61: 731-733.

43. Heinrichs M, Baumgartner T, Kirschbaum C, Ehlert U (2003) Social support and oxytocin interact to suppress cortisol and subjective responses to psychosocial stress. Biol Psychiatry 54: 1389-1398.

44. Miller M, Bales KL, Taylor SL, Yoon J, Hostetler CM, et al. (2013) Oxytocin and vasopressin in children and adolescents with autism spectrum disorders: Sex differences and associations with symptoms. Autism Res 6: 91-102.

45. Molloy CA, Morrow AL, Meinzen-Derr J, Schleifer K, Dienger K, et al. (2006) Elevated cytokine levels in children with autism spectrum disorder. J Neuroimmunol 172: 198-205.

46. Boulanger LM, Shatz CJ (2004) Immune signalling in neural development synaptic plasticity and disease. Nat Rev Neurosci 5: 521-531. 